\title{
Primary Hyperparathyroidism in Pregnancy: A Case Series
}

Naile GOKKAYA, Adem GUNGOR, Arzu BILEN, Habib BILEN

\section{Introduction:}

Primary Hyperparathyroidism (PHP) in pregnancy is very rare condition and the true incidence of PHP in pregnancy is not well known. PHP is associated with significant maternal and fetal morbidity and mortality. PHP is diagnosed based upon levels of blood calcium and parathyroid hormone (PTH) in normal renal function status. However diagnosis of PHT can be difficult, the physiological changes of pregnancy in calcium homeostasis such as maternal blood volume expansion, hypoalbuminemia and increased glomerular filtration rate contribute to the difficulty of PHP diagnosis during pregnancy.

\section{Cases:}

We present four case reports about patients, having PHP in pregnancy. Three of patients, not to be controlled biochemically, denied the parathyroidectomy operation although they are informed about the details of their disease. They are followed up with medical therapy. The first one had no maternal or fetal complications, the second one acquired nephrolithiasis crisis in the last trimester and the third one gave a birth prematurely born baby who succumbed to tetany. The fourth patient who underwent parathyroidectomy operation in the second trimester had no maternal or fetal complications.

\section{Conclusion:}

PHP in pregnancy is a preventable cause of fetal and maternal morbidity and mortality. Suspecting PHP during the pregnancy period and an early diagnosis can provide maternal and fetal well-being. During pregnancy, surgery should be avoided in the first and third trimester and conservative management should be preferred instead. Current evidence supports parathyroidectomy as the main treatment in the second trimester of pregnancy. 